\title{
Local Mosquito-Borne Transmission of Zika Virus - Miami-Dade and Broward Counties, Florida, June-August 2016
}

\begin{abstract}
Anna Likos, $\mathrm{MD}^{1}$; Isabel Griffin, $\mathrm{MPH}^{1}$; Andrea M. Bingham, $\mathrm{PhD}^{1}$; Danielle Stanek, DVM ${ }^{1}$; Marc Fischer, MD²; Stephen White, MS ${ }^{1}$; Janet Hamilton, $\mathrm{MPH}^{1}$; Leah Eisenstein, $\mathrm{MPH}^{1}$; David Atrubin, $\mathrm{MPH}^{1}$; Prakash Mulay, MBBS ${ }^{1}$; Blake Scott, $\mathrm{MPH}^{1}$; Patrick Jenkins, MPH ${ }^{1}$; Danielle Fernandez, $\mathrm{MPH}^{1}$; Edhelene Rico, $\mathrm{MPH}^{1}$; Leah Gillis, $\mathrm{PhD}^{1}$; Reynald Jean, $\mathrm{MD}^{1}$; Marshall Cone, $\mathrm{MPH}^{1}$; Carina Blackmore, $\mathrm{PhD}^{1}$; Janet McAllister, $\mathrm{PhD}^{2}$; Chalmers Vasquez ${ }^{3}$; Lillian Rivera, $\mathrm{PhD}^{1}$; Celeste Philip, $\mathrm{MD}^{1}$
\end{abstract}

On September 23, 2016, this report was posted as an MMWR Early Release on the MMWR website (http://www.cdc.gov/mmwr).

During the first 6 months of 2016, large outbreaks of Zika virus disease caused by local mosquito-borne transmission occurred in Puerto Rico and other U.S. territories, but local mosquito-borne transmission was not identified in the continental United States $(1,2)$. As of July 22, 2016, the Florida Department of Health had identified 321 Zika virus disease cases among Florida residents and visitors, all occurring in either travelers from other countries or territories with ongoing Zika virus transmission or sexual contacts of recent travelers.* During standard case investigation of persons with compatible illness and laboratory evidence of recent Zika virus infection (i.e., a specimen positive by real-time reverse transcription-polymerase chain reaction [rRT-PCR], or positive Zika immunoglobulin $\mathrm{M}$ [IgM] with supporting dengue serology [negative for dengue IgM antibodies and positive for dengue IgG antibodies], or confirmation of Zika virus neutralizing antibodies by plaque reduction neutralization testing [PRNT]) (3), four persons were identified in Broward and Miami-Dade counties whose infections were attributed to likely local mosquito-borne transmission. Two of these persons worked within 120 meters (131 yards) of each other but had no other epidemiologic connections, suggesting the possibility of a local community-based outbreak. Further epidemiologic and laboratory investigations of the worksites and surrounding neighborhood identified a total of 29 persons with laboratory evidence of recent Zika virus infection and likely exposure during late June to early August, most within an approximate 6-block area. In response to limited impact on the population of Aedes aegypti mosquito vectors from initial ground-based mosquito control efforts, aerial ultralow volume spraying with the organophosphate insecticide naled was applied over a 10 square-mile area beginning in early August and alternated with aerial larviciding with Bacillus thuringiensis subspecies israelensis (Bti), a group biologic control agent, in a central 2 square-mile area. No additional cases were identified after implementation of this mosquito control strategy. No increases in emergency department (ED) patient visits associated with

\footnotetext{
*https://wwwn.cdc.gov/nndss/conditions/zika-virus-disease-and-zika-viruscongenital-infection/case-definition/2016/.
}

aerial spraying were reported, including visits for asthma, reactive airway disease, wheezing, shortness of breath, nausea, vomiting, or diarrhea. Local and state health departments serving communities where Ae. aegypti, the primary vector of Zika virus, is found should continue to actively monitor for local transmission of the virus. ${ }^{\dagger}$

\section{Investigations of Two Cases of Isolated Local Transmission of Zika Virus}

As of July 22, 2016, among the 321 cases of Zika virus infection in Florida residents or visitors, Miami-Dade County and neighboring Broward County reported the highest and second highest numbers of cases in Florida (93 and 51, respectively), accounting for $30.4 \%$ and $16.7 \%$ of travel-associated cases in nonpregnant women, respectively.

In early July 2016, an adult female resident of Miami-Dade County (patient A) sought treatment at a local hospital with fever, rash, and arthralgia. Serum and urine specimens, which were collected 3 days after symptom onset, were positive for Zika virus by rRT-PCR. Less than 1 week later, an adult male resident of Broward County (patient B) sought treatment for fever, rash, and arthralgia. Zika virus infection was confirmed by rRT-PCR on a urine specimen and serum IgM by enzyme-linked immunosorbent assay (ELISA) (to minimize the potential for false positives, the Florida Department of Health protocol requires two positive results for index case identification). Investigation of both cases indicated no recent travel to or sexual contact with a recent traveler to an area with active Zika virus transmission, no association with household contacts who recently traveled, and no close personal contact with a patient with confirmed Zika virus infection. There were no epidemiologic links between the two patients, and their residences were separated by $>10$ miles. BG-Sentinel (Biogents AG, Regensburg, Germany) mosquito traps, designed for researchers, collected a limited number of $A e$. aegypti and Ae. albopictus specimens around the patients' residences, and PCR testing of pooled mosquitoes for Zika virus was negative (Sharon Isern, Department of Biological Sciences, Florida Gulf Coast University, personal communication, 2016).

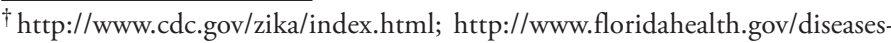
and-conditions/zika-virus/index.html?utm_source=flhealthIndex.
} 
To identify additional evidence of local transmission, household contacts of patients A and B were interviewed regarding recent illness and travel, and specimens were requested for Zika virus testing. Among seven household contacts of the two patients, none reported symptomatic illness and only one had laboratory evidence of recent flavivirus virus infection (Zika virus IgM results and neutralizing antibodies for both Zika virus and dengue, indicating probable Zika virus infection). This person had moved from Haiti to Florida 1 month before onset of symptoms in patient $\mathrm{A}$ and was classified as having a travel-associated case of Zika virus disease.

To identify recent infections in the surrounding neighborhoods of patients $A$ and $B$, systematic surveys were conducted of all households located within 150-300 meters (164-328 yards) of each patient's residence. In addition, an outdoor worksite near patient B's residence also was sampled; these areas were selected based on the typical flight range of Ae. aegypti (4). Surveys were conducted at the end of July and consisted of urine specimen collection and a standardized questionnaire regarding general risk factors. Three visit attempts were made for each occupied residence. Children aged $<5$ years and persons with recent travel to an area with ongoing Zika virus transmission were excluded. Among 116 urine specimens collected from persons from 54 households and one worksite, all were negative for Zika virus by rRT-PCR. In addition, enhanced passive surveillance through syndromic surveillance, review of public health and commercial laboratory results, and notification by local health care providers did not identify any additional cases related to patients $\mathrm{A}$ and $\mathrm{B}$.

\section{Investigations of an Outbreak of Local Transmission of Zika Virus}

On July 9 and July 10, a resident of Miami-Dade County (patient C) and a resident of Broward County (patient D) had onset of fever, rash, and arthralgia; rRT-PCR confirmation of Zika virus infection was obtained approximately 2 weeks after symptom onset. No epidemiologic links between these patients and patients A and B were identified. Although the residences of patients $\mathrm{C}$ and $\mathrm{D}$ were $>20$ miles apart, their workplaces were located within 120 meters of each other in MiamiDade County, in a mixed-use neighborhood with residences, businesses, and restaurants. Workplace A (the workplace of patient C) was entirely enclosed with air conditioning, but sites for mosquitoes to lay eggs (mosquito larval development sites) were observed in close proximity to the workplace, including an outdoor break area. Investigation of workplace A identified no other symptomatic employees, and no other employees were tested. However, patient $\mathrm{C}$ reported having a symptomatic customer. The customer had a positive Zika IgM test, indicative of presumptive recent $\mathrm{Zika}$ virus infection;
PRNT results are pending. Workplace B (the workplace of patient D) was primarily open air with only a small, enclosed area with air conditioning. Standing water was abundant and multiple mosquito larval development sites were identified on the property. Initial questioning of workplace B employees identified 17 employees with illness compatible with Zika virus infection, 15 of whom provided serum and urine specimens; three employees had rRT-PCR or serologic confirmation of Zika virus infection. In addition, 14 asymptomatic employees consented to provide either urine or serum specimens; two were serologically confirmed to be infected with Zika virus.

To determine whether an outbreak was occurring in the wider community, a survey was conducted among 96 households within a 150 -meter radius (approximately 6 blocks) of the two workplaces during July 28-30. Three visit attempts were made for each occupied residence in the area. Children aged $<5$ years and persons with recent travel to a Zika-affected area were excluded. Of the 96 approached households, 52 urine specimens were collected from 28 households; six persons tested positive for Zika virus by rRT-PCR, all of whom were asymptomatic. Based on these results, on August 1, CDC issued a health advisory notice, recommending that pregnant women avoid nonessential travel to a 1 square-mile area that included the 6-block area of concern plus a wide buffer zone (5) (Figure 1).

To investigate whether active transmission was occurring beyond the 6-block area, three additional surveys were conducted at locations bordering the edges of the 1 square-mile area. In the northwest corner survey, 247 households were approached, and 142 urine specimens were collected from 73 households, one of which was rRT-PCR-positive. Local transmission was ruled out in this case, because the specimen was collected from a person who recently returned from a Zikaaffected country. In the second border survey, 127 households were approached, and 102 urine specimens were collected from 50 households. The third border survey approached 68 households, and 45 urine specimens were collected from 27 households. None of the specimens collected from the second or third surveys tested positive by rRT-PCR.

In early August, the health department partnered with a federally qualified health center in the 1 square-mile area to establish a Zika clinic to identify additional infections. The clinic offered testing to persons who lived or worked in the affected area. Three of the 77 urine specimens collected from patients at this local clinic were positive by rRT-PCR. The three patients with infection were all symptomatic and had possible exposure within or adjacent to the initial 6-block area: one patient worked in the area, another was a contact of a workplace $\mathrm{B}$ employee who also frequented the area, and the third 
FIGURE 1. One square-mile area encompassing the 6-block area of the Zika virus transmission outbreak, plus a buffer zone - Miami-Dade County, Florida, July-August 2016

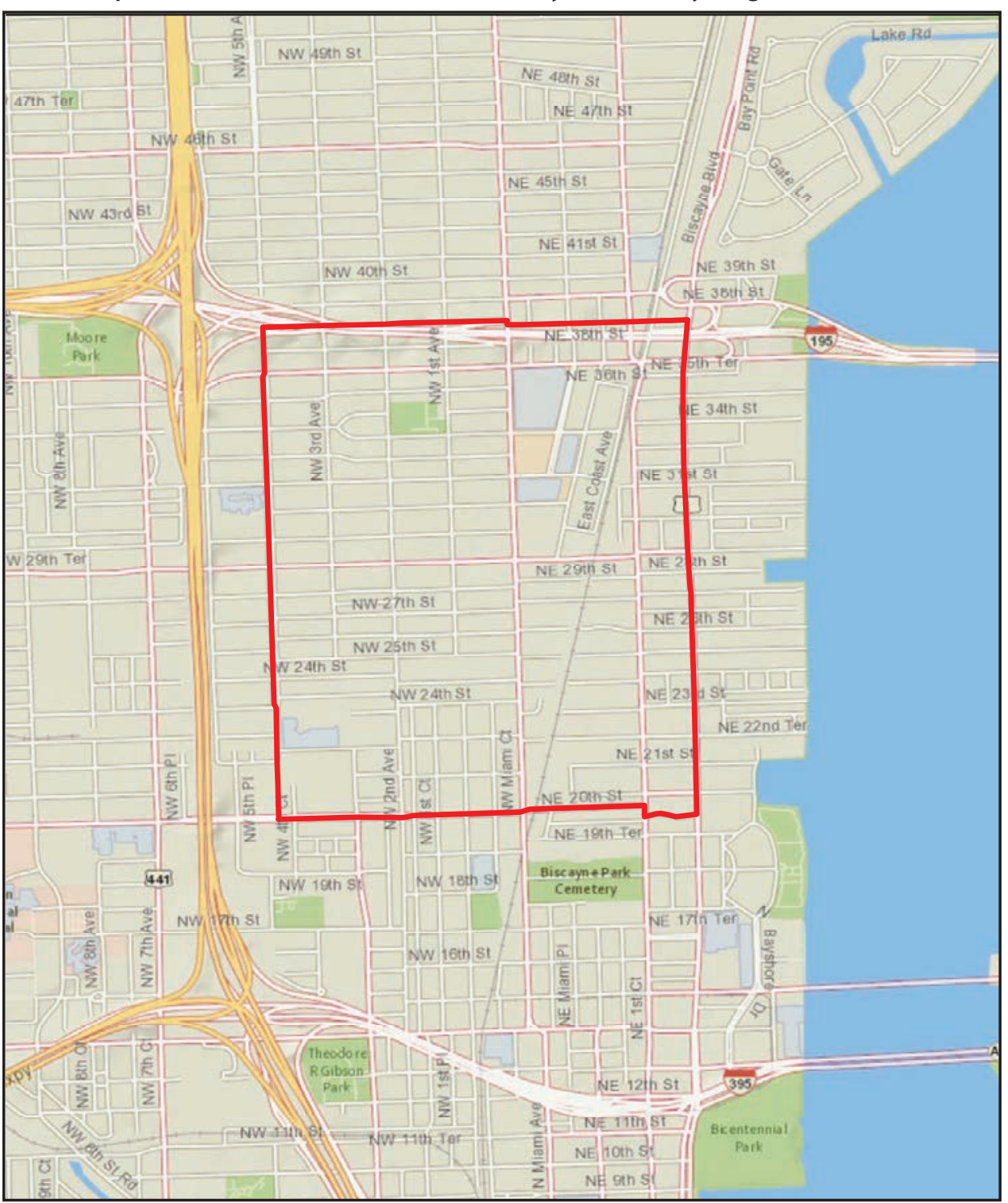

was a customer of another business in the area (workplace $\mathrm{C}$ ), which was located within 150 meters of workplaces A and B.

At the request of the employer at workplace $\mathrm{C}$, testing was offered to workplace $\mathrm{C}$ employees. Workplace $\mathrm{C}$ was an entirely open air workplace, with multiple mosquito larval development sites in close proximity to the workplace. Among 90 employees, serum and urine specimens were collected from five who had a history of Zika-compatible symptoms; an additional three symptomatic employees and 55 asymptomatic employees provided urine specimens only. Three of the 63 employees tested were rRT-PCR-positive, and two additional employees had serologic evidence of infection. All five reported symptoms.

As of September 1, an additional seven symptomatic persons with laboratory evidence of recent Zika virus infection had been reported from the 1 square-mile area: a second customer of workplace $\mathrm{C}$, three residents of the area, and three persons who work in the area. Onset of illness for all 23 symptomatic persons ranged from June 30 to August 5 (Figure 2). Overall, epidemiologic and laboratory investigations of the workplaces and surrounding neighborhood ${ }^{\S}$ identified 29 persons with laboratory evidence of recent Zika virus infection and likely exposure within an approximate 6-block area.

\section{Investigations Related to Mosquito Control Strategies and Implementation}

Mosquito trapping in the area with ongoing local transmission identified large numbers of Ae. aegypti females as well as a large number of mosquito larval development sites. Initial control efforts, including eliminating standing water, larviciding, and applying insecticides by backpack and truck-mounted spraying to control adult mosquitoes, were later augmented by aerial spraying. On July 23 , the day after the workplace exposure link was established, door-to-door inspections and backpack spraying commenced with the pyrethroid insecticides sumithrin/ prallethrin in the 6-block core area. The following day, these activities were augmented by truck-mounted spraying with the pyrethroid insecticides sumithrin/prallethrin, permethrin, and deltamethrin over the larger 1-square-mile area. Mosquito trapping produced an average of 24, 27, and 23 female Ae. aegypti per trap per day on 3 successive days within 5-7 days of initiating control efforts (Figure 3). None of the mosquito pools tested for Zika virus were positive (Sharon Isern, Department of Biological Sciences, Florida Gulf Coast University, personal communication, 2016).

Because of persistently high numbers of trapped Ae. aegypti females, aerial ultralow volume spraying with naled, an organophosphate insecticide used to kill adult mosquitoes, was applied over a 10 -square-mile area beginning August 4, and alternated with aerial larviciding with $\mathrm{Bti}$ in a central 2-square-mile area

\footnotetext{
\$ http://www.cdc.gov/zika/pdfs/zika-draft-interim-conus-plan.pdf.
} 
FIGURE 2. Number of patients $(n=23)$ with symptomatic cases of locally transmitted Zika virus infection, by week of symptom onset and outbreak status - Miami-Dade and Broward counties, Florida, June-August 2016

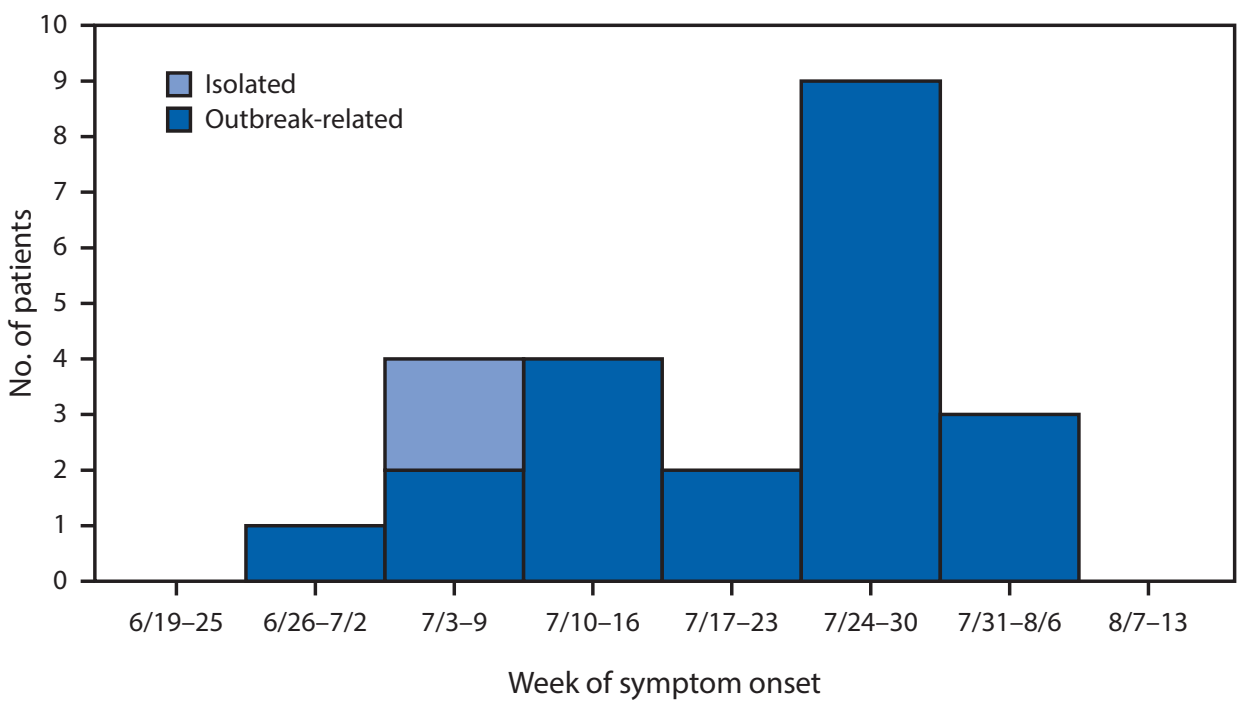

remained the same, and the percentage of ED visits for these four syndromes, when compared week by week, was similar to the same period during the 2 previous years. ED patient visits for nausea, vomiting, or diarrhea also did not increase. Review of historical insecticiderelated illness and injury cases from state surveillance data for January 1, 2001, through August 17, 2016, identified one probable and 45 suspected cases** related to naled exposure in Florida; the last case was reported in July 2013.

\section{Discussion}

The first identified occurrence of isolated local mosquito-borne transmission and the first identified outbreak of mosquito-borne Zika virus infection in the continental United States occurred around the area with ongoing local transmission. To minimize potential human effects, spraying occurred during the early morning hours when fewer persons were outside, and this also limited effects on non-target organisms such as bees. Female Ae. aegypti counts decreased to one per trap per day after the second aerial spraying with naled; counts then gradually returned to high levels ( $>20$ per trap per day) in the adulticide-only spray area, but were maintained at about 5-10 per trap per day for at least 1 month in the area treated with both adulticide and larvicide (Figure 3).

In addition, to reduce the number of larval development sites, the Florida Department of Health in Miami-Dade County reemphasized its ongoing Drain and Cover campaign, 5 encouraging residents to remove or cover standing water around their homes and businesses.

To address public health and community concerns about the use of naled aerial adulticiding, calls to poison information centers and ED visits were monitored in the days following treatments. Seven exposure calls were reported to the Florida Poison Information Center, four of which reported symptoms (vomiting, eye irritation, dizziness, vertigo, and edema). Data captured by the Electronic Surveillance System for the Early Notification of Community-based Epidemics-FL indicated that total ED patient visits by persons residing in the four zipcode areas with spraying were unchanged from the number of visits before spraying. In addition, patient visits for asthma, reactive airway disease, wheezing, or shortness of breath

\footnotetext{
http://www.floridahealth.gov/diseases-and-conditions/mosquito-bornediseases/prevention.html.
} in Florida in Miami-Dade and Broward counties during June-August, 2016. After identification of two cases linked geographically by places of employment, enhanced passive and active case finding identified a cluster of 29 infections with illness onset during June 30-August 5. Multiple cases were identified in residents of the affected area; however, the investigation highlighted the potential risk for workplace mosquito exposure. Workplaces A, B, and C all had significant open-air areas where employees worked or took breaks and which were in close proximity to identified larval development sites. Health departments should collect information on occupation, industry, and workplace as part of ongoing Zika case investigations. Including the systematic collection of this information as part of surveillance might facilitate identifying future workplace-associated outbreaks.

Aggressive mosquito control efforts, including aerial adulticiding and larviciding, most likely contributed to a decrease in Zika virus transmission; no new cases in this area were identified with symptom onset more than 2 weeks after the first aerial adulticide and larvicide applications. The affected community also played a role in preventing new infections when residents and businesses began observing Drain and Cover prevention measures. Although the outbreak continued for more than 1 month, it remained limited to a small geographic area, as has been the case in previous arbovirus outbreaks in Florida (G).

Despite intensive investigation, no evidence of further spread was identified within the households or neighborhoods of two unrelated locally transmitted cases. Epidemiologic

** http://www.cdc.gov/niosh/topics/pesticides/pdfs/casedef.pdf. 


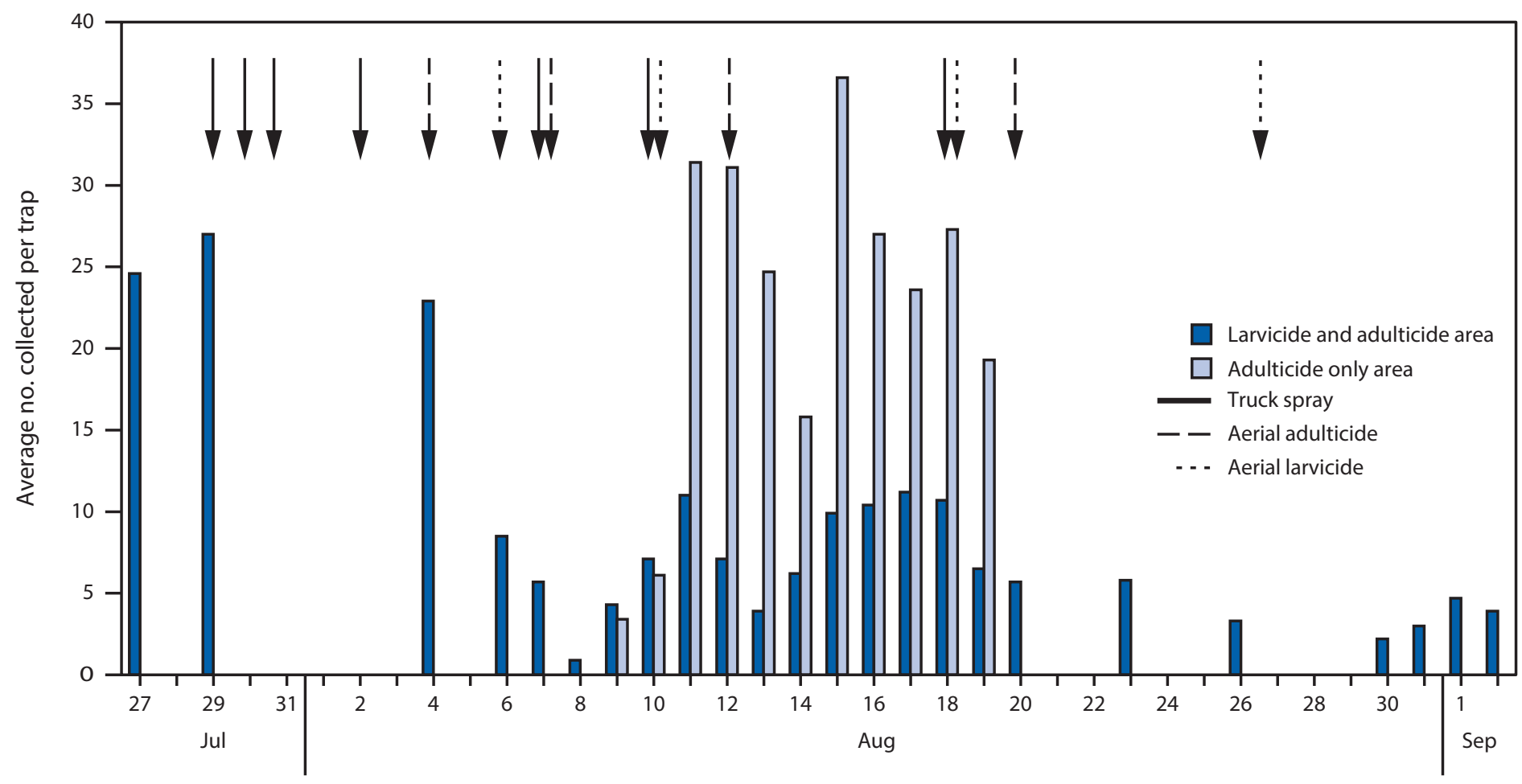

Date/Month

investigations of outbreaks of dengue, a related flavivirus spread by the same vectors, suggest that the wide use of air conditioning and low population densities limit spread of viruses transmitted by Ae. aegypti, a mosquito that bites indoors and outdoors and has a limited flight range ( 7 ); however, other factors might play a role in limited spread. Open doors and windows were observed at the homes of both Patients A and $\mathrm{B}$, but air conditioning appeared functional in neighboring houses, and population density thresholds for flavivirus transmission have not been determined.

Currently, the Food and Drug Administration's Emergency Use Authorization recommends rRT-PCR testing of urine and serum. ${ }^{\dagger \dagger}$ However, many assessments of ongoing community transmission in these investigations included collection of urine specimens only for rRT-PCR testing. This approach had several advantages. For example, a positive PCR test provides a definitive diagnosis of Zika virus infection, no phlebotomist and fewer laboratory supplies are required (reducing costs and required skills for investigations), and willingness of survey respondents to provide a single, noninvasive specimen might have enhanced participation. In addition, detection of Zika virus RNA has been documented with a higher frequency and for a longer duration in urine than in serum (8). However, a

$\overline{\dagger \dagger}$ http://www.cdc.gov/zika/laboratories/types-of-tests.html. disadvantage of only collecting urine is that persons with earlier exposures might no longer have viral RNA present in their urine, and without serologic confirmation, a diagnosis of Zika virus infection could be missed.

Control of Ae. aegypti during outbreaks is hampered by factors including a large number of cryptic larval development sites in urban environments, the possibility that truck-based spraying might not reach backyards or areas distant from roads, and the presence of adult mosquitoes indoors. In this affected area, high numbers of Ae. aegypti adults persisted despite aggressive efforts at eliminating larval development sites and truck-based and backpack spraying with adulticides. In contrast, mosquito counts decreased $>10$ fold following two aerial applications of naled at 3-day intervals; however, a sustained reduction was maintained only in the area sprayed aerially with both naled and Bti. Substantial reductions in mosquito counts coincided with apparent cessation of the outbreak.

Aerial insecticide applications have the potential to treat large areas rapidly and more uniformly; however, data on the efficacy of controlling Ae. aegypti populations by aerial spraying with modern ultralow volume spray technologies that can precisely control droplet size are limited. Less than one ounce of naled per acre is used for aerial spraying, which might explain the absence of observed negative health effects during and after aerial spraying. This finding is consistent with previous reports 


\section{Summary}

What is already known about this topic?

During the first half of 2016, large outbreaks of Zika virus infection caused by local mosquito-borne transmission of Zika virus occurred in many countries in Latin America and the Caribbean in Puerto Rico and other U.S. territories, but local mosquito-borne transmission was not identified in the continental United States.

What is added by this report?

During June 30-August 5, 2016, the first recognized outbreak of mosquito-borne transmission of Zika virus in the continental United States occurred in a neighborhood in Miami-Dade County, Florida. Twenty-nine persons with Zika virus infection had likely exposure within an approximate 6-block area. The outbreak ended after aerial spraying to control mosquitoes. No increases in short-term health effects were associated with spraying.

What are the implications for public health practice?

To reduce the risk for local Zika virus transmission within the continental United States, persons returning to the continental United States from areas with ongoing Zika virus transmission should use daily mosquito repellent for 3 weeks and follow CDC published guidance to prevent sexual transmission of Zika virus. Investigation of reported cases by local and state health departments provides opportunities to control Zika virus outbreaks within the continental United States. Jurisdictions with Aedes aegypti present should ensure ongoing capacity for comprehensive mosquito control.

showing no difference in naled metabolites in urine before and after spraying, suggesting that residents in spray zones have negligible insecticide exposure $(9,10)$.

The findings in this report are subject to at least four limitations. First, the number of persons infected with Zika virus likely was higher than reported. Most persons identified with Zika virus infection did not seek medical care; several were asymptomatic and were only identified by investigations of workplaces and neighborhood surveys. Second, the neighborhood surveys in the outbreak area were a convenience sample selected to detect ongoing transmission, and thus, the proportions of persons identified with recent infection could not be extrapolated to produce communitywide estimates of infection incidence. No other similar investigations exist for comparison of findings. Third, some persons infected earlier in the course of the outbreak might not have had Zika virus RNA still present in urine, resulting in an underestimation of the number of infected persons among those surveyed. Finally, the threshold reduction of Ae. aegypti populations needed to interrupt Zika virus transmission in South Florida is unknown and likely would vary by location and environment. Thus, although the combination of aerially applied naled and Bti along with source reduction and ground-based applications of larvicide and adulticides reduced Ae. aegypti populations to low levels, it cannot be concluded definitively that these reductions were responsible for ending the outbreak.

Local and state health departments serving communities with a competent Zika virus vector should continue active monitoring for local virus transmission. To reduce risk for local transmission within the continental United States, persons returning from areas with ongoing Zika virus transmission should use insect repellent routinely for 3 weeks after return to prevent human-to-mosquito-to-human transmission and should use condoms to prevent sexual transmission. ${ }^{\$ \$}$ All residents, regardless of travel history, and all business establishments should empty or drain standing water around their homes and businesses. Clinicians who suspect Zika virus disease in patients who reside in or have recently returned from areas with ongoing Zika virus transmission should consider testing for Zika virus and promptly report cases to public health officials. Clinicians in areas where the vector is found might consider testing persons with compatible illness even in the absence of travel.

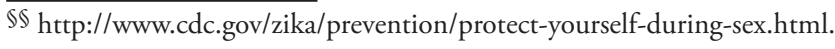

\section{Acknowledgments}

Epidemiology, laboratory, and public health preparedness personnel; local hospital and clinic partners in Miami-Dade County; CDC CERT members and headquarters staff members; Florida Medical Reserve Corp volunteers; Broward, Miami-Dade, Lee, Manatee County and the Keys mosquito control districts; the Healthy Start Coalition of Miami-Dade County, Inc; Florida Department of Agriculture and Consumer Services; Sharon Isern, Florida Gulf Coast University; the American Congress of Obstetrics and Gynecologists District XII; the Florida Poison Control Center.

\footnotetext{
${ }^{1}$ Florida Department of Health; ${ }^{2}$ Division of Vector-Borne Diseases, National Center for Emerging and Zoonotic Infectious Diseases, CDC; ${ }^{3}$ Miami-Dade County Mosquito Control District, Florida.

Corresponding author: Anna Likos, anna.likos@flhealth.gov, 850-245-4111.
}

\section{References}

1. Armstrong P, Hennessey M, Adams M, et al. Travel-associated Zika virus disease cases among U.S. residents-United States, January 2015February 2016. MMWR Morb Mortal Wkly Rep 2016;65:286-9. http://dx.doi.org/10.15585/mmwr.mm6511e1

2. Adams L, Bello-Pagan M, Lozier M, et al. Update: ongoing Zika virus transmission-Puerto Rico, November 1, 2015-July 7, 2016. MMWR Morb Mortal Wkly Rep 2016;65:774-9. http://dx.doi.org/10.15585/ mmwr.mm6530e1

3. Rabe IB, Staples JE, Villanueva J, et al. Interim guidance for interpretation of Zika virus antibody test results. MMWR Morb Mortal Wkly Rep 2016;65:543-6. http://dx.doi.org/10.15585/mmwr.mm6521e1

4. Harrington LC, Scott TW, Lerdthusnee K, et al. Dispersal of the dengue vector Aedes aegypti within and between rural communities. Am J Trop Med Hyg 2005;72:209-20. 
5. CDC. Guidance for travel and testing of pregnant women and women of reproductive age for Zika virus infection related to the investigation for local mosquito-borne Zika virus transmission in Miami-Dade and Broward counties, Florida. Atlanta, GA: US Department of Health and Human Services, CDC; 2016. https://emergency.cdc.gov/han/han00393.asp

6. CDC. Locally acquired dengue-Key West, Florida, 2009-2010. MMWR Morb Mortal Wkly Rep 2010;59:577-81.

7. Reiter P, Lathrop S, Bunning M, et al. Texas lifestyle limits transmission of dengue virus. Emerg Infect Dis 2003;9:86-9. http://dx.doi. org/10.3201/eid0901.020220
8. Bingham AM, Cone M, Mock V, et al. Comparison of test results for Zika virus RNA in urine, serum, and saliva specimens from persons with travelassociated Zika virus disease-Florida, 2016. MMWR Morb Mortal Wkly Rep 2016;65:475-8. http://dx.doi.org/10.15585/mmwr.mm6518e2

9. CDC. Human exposure to mosquito-control pesticides-Mississippi, North Carolina, and Virginia, 2002 and 2003. MMWR Morb Mortal Wkly Rep 2005;54:529-32.

10. Duprey Z, Rivers S, Luber G, et al. Community aerial mosquito control and naled exposure. J Am Mosq Control Assoc 2008;24:42-6. http:// dx.doi.org/10.2987/5559.1 